TTR

Traduction, terminologie, re?daction

\title{
Étude comparative des combinaisons lexicales dans deux domaines du savoir (aéronautique et philosophie) et opposition entre les collocations lexicales et conceptuelles
}

\section{Claudine Bertrand}

Volume 11, numéro 1, 1er semestre 1998

Diachronie et synchronie

Diachronics and Synchronics

URI : https://id.erudit.org/iderudit/037323ar

DOI : https://doi.org/10.7202/037323ar

Aller au sommaire du numéro

Éditeur(s)

Association canadienne de traductologie

ISSN

0835-8443 (imprimé)

1708-2188 (numérique)

Découvrir la revue

Citer cet article

Bertrand, C. (1998). Étude comparative des combinaisons lexicales dans deux domaines du savoir (aéronautique et philosophie) et opposition entre les collocations lexicales et conceptuelles. TTR, 11(1), 229-249.

https://doi.org/10.7202/037323ar

\section{Résumé de l'article}

Étude comparative des combinaisons lexicales dans deux domaines du savoir (aéronautique et philosophie) et opposition entre les collocations lexicales et conceptuelles - Notre étude porte sur la combinatoire lexicale en langue de spécialité et cherche à déterminer l'organisation lexicale, syntaxique et sémantique liée à une contrainte d'usage sous-jacente à deux domaines spécifiques - l'aéronautique et la philosophie. Une analyse des points de vue des chercheurs en langue générale et en langue de spécialité sera présentée en première partie. Ensuite, nous ferons la distinction entre les collocations lexicales et les collocations conceptuelles en langue de spécialité. Enfin, nous présenterons la méthodologie utilisée pour mener à bien notre mémoire de maîtrise (en cours) ainsi que des résultats fragmentaires, l'objectif du mémoire étant a) de relever les différents types de combinaisons $(\mathrm{T}+\mathrm{V}, \mathrm{V}+\mathrm{T}, \mathrm{T}+\mathrm{adj}$, adj $+\mathrm{T}, \mathrm{T}+$ prép $+\mathrm{N}, \mathrm{N}+$ prép $+\mathrm{T}$ ); b) de distinguer les collocations lexicales et conceptuelles, s'il en est, et c) d'étudier les tendances de comportement dans l'un et l'autre domaine.
Tous droits réservés (C) TTR: traduction, terminologie, rédaction — Les auteurs, 1998
Ce document est protégé par la loi sur le droit d'auteur. L’utilisation des services d'Érudit (y compris la reproduction) est assujettie à sa politique d'utilisation que vous pouvez consulter en ligne. 


\title{
Étude comparative des combinaisons lexicales dans
} deux domaines du savoir (aéronautique et philosophie) et opposition entre les collocations lexicales et conceptuelles

\author{
Claudine Bertrand
}

\section{Introduction}

Depuis quelques années, les combinaisons lexicales font l'objet de multiples études. De nombreux spécialistes de la langue - de Haussmann à L'Homme, en passant par Mel'čuk, Heid, Freibott, Kocourek, Sager, Meyer, Martin, Cohen, Pavel, Benson, Lainé -, pour $n^{\prime}$ en nommer que quelques-uns, ont tenté, et tentent encore, de circonscrire le phénomène de la combinatoire lexicale. La problématique est de taille. En effet, il existe toutes sortes d'associations de mots, fortuites ou lexicalisées, figées ou semi-figées, et la langue française foisonne de tels groupements : il peut s'agir de " mots composés " - bloc-moteur, eau-de-vie; de * syntagmes libres " témoin détecteur d'incendie (Kocourek; 1982) de * syntagmes terminologiques " (ou " termes complexes n) - eau lourde, centre de gravité (Kocourek; 1982); de groupements privilégiés - satvegarder 
un fichier (L'Homme et Gemme; 1997). En outre, leur découpage présente de nombreuses difficultés, notamment la délimitation des unités proprement terminologiques telles qu'elles sont définies par les terminologues.

La présente recherche s'inscrit dans le cadre d'un projet de mémoire de maîtrise qui porte sur l'étude des groupements lexicaux " privilégiés " de la langue spécialisée, c'est-à-dire des groupes composés de deux lexèmes utilisés selon une certaine convention au sein d'une communauté de spécialistes d'un domaine donné et dont l'un des constituants est un * terme ". Rappelons qu'un * terme " (simple ou complexe), tel qu'il est défini dans la norme ISO 1087, est une désignation au moyen d'une unité linguistique d'une notion définie dans une langue de spécialité.

Les chercheurs (terminologues, linguistes, lexicographes ou autres professionnels langagiers) ont recours à diverses dénominations pour désigner ce type de groupement privilégié : collocation (Mel'čuk et al.; 1995), phraséologisme (Pavel; 1994), cooccurrents (Cohen; 1986), combinaison lexicale (L'Homme; 1996). Notre propos n'est pas de nous pencher sur ces variantes; aussi, pour les besoins de notre recherche, avons-nous adopté la terminologie proposée par L'Homme (1998), à savoir CLS (combinaison lexicale spécialisée) pour les groupements de la langue spécialisée et collocation pour ceux de la langue commune.

Dans le cadre de notre mémoire, nous avons choisi d'examiner les CLS appartenant à deux domaines de spécialité qui nous semblaient suffisamment distincts pour pouvoir en observer les différences de comportement lexical. En effet, l'un, l'aéronautique, est associé aux sciences dites exactes, et l'autre, la philosophie, est associé aux sciences humaines. Toutefois, notre mémoire n'étant pas encore terminé, nous désirons souligner que nous ne présenterons ici que des résultats fragmentaires.

\section{État de la question}

Nous avons examiné, dans un premier temps, comment différents auteurs definissent la collocation en langue commune et la CLS en 
langue de spécialité, pour en venir, dans un deuxième temps, et de manière plus spécifique, à la notion de collocation lexicale par opposition à la collocation conceptuelle.

\section{Point de vue de la langue commune}

Les travaux de Haussmann (1979) s'inscrivent dans le cadre de la lexicographie. L'auteur s'intéresse particulièrement au recensement des collocations et à l'encodage de celles-ci dans des outils tels les dictionnaires. Pour Haussmann, la collocation se compose de deux éléments appelés base et collocateur, et leur relation est qualifiée de " polaire ": la base conserve son sens (une sémantique autonome), contrairement au collocateur (sémantiquement dépendant), qui ne s'interprète qu'en cooccurrence avec la base (par ex., célibataire endurci). Il existe donc une contrainte lexicale pour ce deuxième élément. Haussmann ajoute qu'il existe une " affinité " entre les constituants de la collocation, ce qui entraine un constat d'imprévisibilité quant au choix des collocateurs, d'où la nécessité de mémoriser les collocations.

Pour leur part, Benson et al. (1986) ont publié un dictionnaire (unilingue anglais) qui met l'accent sur la combinatoire des entrées. Dans cet ouvrage, les auteurs définissent la collocation comme étant une combinaison de mots récurrente et semi-figée. Ils répartissent les collocations en deux groupes, soit les collocations grammaticales (admiration for) et les collocations lexicales (world championship) qui sont des combinaisons à lexèmes. À l'instar de Haussmann, Benson et al. reconnaissent que les collocations de ce type sont arbitraires et imprévisibles, et donc qu'il est nécessaire pour les non-natifs de la langue de les apprendre.

Mel'čuk et al. $(1984,1995)$ ont élaboré un modèle (sens-texte) qui permet de décrire de façon systématique les expressions lexicales plus ou moins figées de la langue générale, expressions idiomatiques ou non mais de caractère conventionnel, que tout locuteur non natif de la langue doit apprendre s'il désire communiquer de manière juste en utilisant les " bonnes tournures " propres à une langue donnée. Pour Mel'cuk et al., les collocations sont des groupes * ... lexicalement contraints, c'est-à-dire dont la combinatoire n'est déterninée ni par leur 
sémantisme ni par leurs propriétés syntaxiques * (Mel'čuk et al. 1995:126). Par exemple, pourquoi, se demandent les auteurs, peut-on dire avoir recours à et non *faire recours à? Aucune règle sémantique ou syntaxique ne force l'emploi d'une expression de préférence à l'autre. Les collocations ne sont donc pas prévisibles. Les auteurs classent les collocations en fonction du rapport sémantique existant entre leurs composants, et ils les présentent d'après ce qu'ils nomment des fonctions lexicales (FL), lesquelles constituent une constante sémantique : $f(x)=y$, dans laquelle $* f$ $x$ constitue la fonction exprimée; $\alpha X$ " l'argument (le mot clé) et * $\mathrm{y}$ * la valeur, c'est-à-dire la résultante.

\section{Exemples : $\quad$ 1. Magn (refuser) $=$ absolument, catégoriquement \\ 2. Magn (peur) = bleue}

Dans ces exemples :

la constante sémantique $=$ intensification (sens 'très')
$\begin{array}{ll}\text { la fonction lexicale }=\text { Magn } \\ \text { le mot clé }= & \text { 1. refuser } \\ \text { la valeur }= & \text { 2. peur } \\ & \text { 1. absolument, catégoriquement } \\ & \text { 2. bleue }\end{array}$

Ainsi, pour Haussmann, Benson et al. de même que Mel'čuk et al., il appert que la collocation se compose de deux éléments (l'un est appelé base par Haussmann; mot clé par Mel'cuk et al.; et l'autre collocateur) et que leur relation est de nature conventionnelle et imprévisible. De plus, les éléments qui composent la collocation sont soumis à une certaine contrainte et la collocation est partiellement compositionnelle, c'est-à-dire que l'un des éléments - le mot clé - garde son sens propre, tandis que son collocateur est lexicalement contraint.

\section{Point de vue de la langue spécialisée}

Cohen (1986) a produit le Lexique de cooccurrents de la Bourse et de la conjoncture économique, dans lequel elle a consigné des associations de mots qu'elle qualifie de " cooccurrences lexicales " (accélération rapide, souscrire une action). Cette auteure s'est inspiré d'un mécanisme s'apparentant au modèle des fonctions lexicales élaboré par Mel'čuk et al. pour classer les cooccurrences en fonction des phases du cycle économique (début, croissance, déclin et fin) et regrouper les 
termes par catégories grammaticales (nom, verbe/sujet, verbe/objet et adjectif). Ses travaux montrent qu'il est possible de systématiser les relations sémantiques entre termes et cooccurrents et qu'un formalisme comme les FL est utilisable pour en rendre compte.

Pavel (1994) a publié un ouvrage dans lequel elle présente une typologie de ce qu'elle nomme les phraséologismes qu'elle définit comme suit : « le phraséologisme LS est une combinaison préférentielle ou une solidarité lexicale d'un terme, appelé noyau ou base, et de mots qui cooccurrent dans la même phrase * (1994:4). Cette auteure distingue 1) la combinatoire syntagmatique liée à la séquence horizontale des cooccurrents (arête + change + de visibilité), et 2) la combinatoire paradigmatique, qui liste des cooccurrents de même catégorie grammaticale pouvant apparaître dans une position déterminée, un à la fois (l'arête + change d'état/compose des facettes) (1994:19). Elle prend également en compte le facteur de commutabilité des éléments pour regrouper les combinaisons en trois catégories soit, 1) combinaison lexicalement figée (promulguer une loi) (1994:7); 2) combinaison à commutabilité restreinte (déformer par, sous translation) (1994:7); et 3) combinaison libre (l'agrégat adopte une configuration, grossit) (1994:7). Les travaux de nature pragmatique de Pavel mettent en lumière la complexité liée à l'élaboration d'une typologie structurelle des combinaisons lexicales qui tienne compte de l'organisation lexicale, syntaxique et sémantique des combinaisons.

\section{Collocations lexicales c. collocations conceptuelles}

Martin (1992) décrit les CLS, qu'il appelle collocations, comme étant des groupements de mots lexicalement contraints, c'est-à-dire des combinaisons lexicales non libres présentant un comportement souvent compositionnel. Il avance également que certaines combinaisons sont susceptibles de généralisation et qu'elles sont donc de nature conceptuelle (par ex, maladie infectieuse, dans laquelle maladie peut accepter des cooccurrents sémantiquement apparentés à infectieuse).

Heid (1992; 1994), à l'instar de Martin, affirme que le niveau conceptuel revêt une importance particulière et qu'il est possible de prévoir des combinaisons lorsque l'on se trouve en présence d'une description conceptuelle détaillée. Il estime qu'il y a plus de chances de 
prévoir les combinaisons en langue de spécialité étant donné que la terminologie de beaucoup de domaines est moins polysémique que dans la langue générale. Selon cet auteur, il existerait une prédominance des combinaisons conceptuelles dans les domaines spécialisés en raison de la spécificité liée à la dénomination de notions concrètes. Heid a étudié, entre autres, le Lexique de cooccurrents de la Bourse et de la conjoncture économique (Cohen; 1986) dans lequel il a relevé les noms ayant en commun certaines propriétés. Il a remarqué, par exemple, que les noms dénotant une " augmentation " ou une * diminution "- hausse, baisse, mouvement, progression, recul, etc. - partageaient, d'une part, pour le concept d' ' augmentation i les verbes : s'amplifier, s'accélérer, s'accentuer et, d'autre part, pour le concept de * diminution » les verbes : ralentir, limiter, freiner. Cette constatation a conduit Heid à mettre en relation le niveau de description sémantique et conceptuel et le comportement collocationnel, soulignant le caractère conceptuel de certains ensembles et sous-ensembles de notions.

Par ailleurs, les recherches de L'Homme $(1995,1996,1997)$ se portent particulièrement sur le recensement et la consignation des CLS dans des outils informatisés. Pour ce faire, l'auteure a étudié et retenu les propriétés et caractéristiques formulées par d'autres spécialistes, à savoir le caractère conventionnel des groupements, leur forme, leur nature compositionnelle ou non compositionnelle, la possibilite de généraliser les bases ou mots clés à des ensembles plus vastes d'unités lexicales et les possibilités de généraliser des relations sémantiques entre les éléments des groupements. Ses travaux ont porté notamment sur l'encodage des combinaisons lexicales en langue de spécialité basé sur une hiérarchie de concepts génériques, l'accent étant ainsi mis sur les relations de type conceptuel de ces combinaisons (de type verbe + nom ou terme; déverbal + préposition + nom ou terme).

Dans le domaine informatique, par exemple, L'Homme et Gemme (1997) ont imaginé un cođage des classes conceptuelles auxquelies appartiennent les termes pour établir une base de données comprenant des enregistrements terminologiques et des enregistrements verbaux, dont le tableau ci-dessous présente un exemple (tiré de l'article Modele d'accès informatisé aux combinaisons lexicales spécialisées, L'Homme et Gemme:1997). 


\begin{tabular}{llll}
$\begin{array}{l}\text { ENREGISTREMENT TERMINOLOGIQUE } \\
\text { Entry }\end{array}$ & \multicolumn{2}{c}{ ENREGISTREMENT VERBAL } \\
Definition file & $\begin{array}{l}\text { A file containing } \\
\text { data records } \\
\text { fichier de données }\end{array}$ & $\begin{array}{l}\text { Entry } \\
\text { Structure }\end{array}$ & $\begin{array}{l}\text { delete } \\
\text { V }+0\end{array}$ \\
Definition & $\begin{array}{l}\text { To remove something } \\
\text { that has previously } \\
\text { been written } \\
\text { or stored. }\end{array}$ \\
Définition & $\begin{array}{l}\text { Fichier qui comporte un } \\
\text { ensemble de données }\end{array}$ & Subject & PEOPLE \\
Domaine & informatique & Object & ENT REP \\
Concept & ENT REP & $\begin{array}{l}\text { Entrée } \\
\text { effacer }\end{array}$ \\
& & Structure & V+O \\
& & Définition & $\ldots$ \\
Sujet & PEOPLE \\
& & Objet & ENT REP
\end{tabular}

Dans cet exemple, l'entrée data file entre dans une classe conceptuelle regroupant des objets a destinés à diffuser ou à contenir de l'information ". Ces unités sont étiquetées comme des representational entities (ENT REP). Quant au verbe effacer, on indique qu'il peut admettre des sujets appartenant à la classe des animés humains (PEOPLE, par exemple, user, programmer). Le verbe admet également des objets appartenant à la classe des representational entities. $\mathrm{Ce}$ modèle permet ainsi de repérer la combinaison $\propto$ effacer fichier de données " ou bien la combinaison " programmeur efface (fichier de données) ».

\section{Méthodologie de notre étude}

\section{Processus de recherche}

Le diagramme de cheminement ci-après illustre les différentes étapes que nous avons suivies. 
Tableau I : Cheminement de recherche

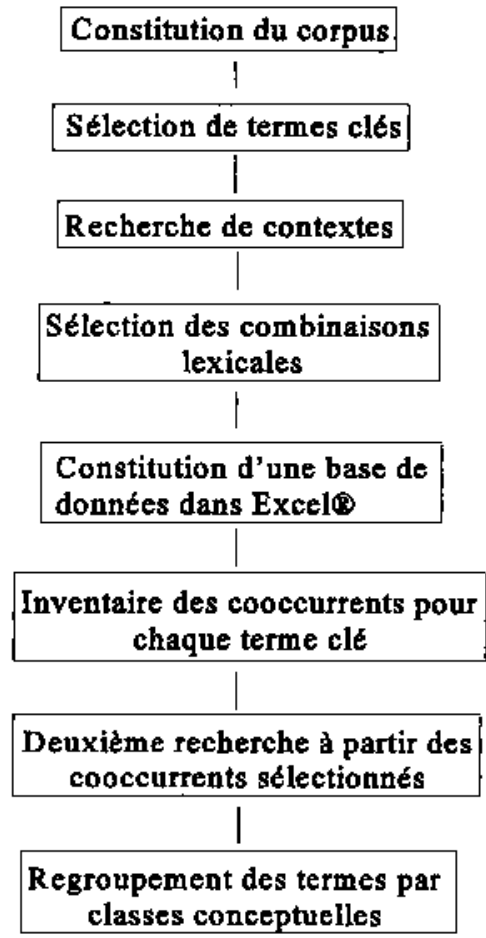

Notre corpus se compose de textes extraits d'ouvrages de formation et de nature normative, liés au contrôle aérien et au pilotage pour le domaine de l'aéronautique, et d'ouvrages présentant les grandes lignes de la pensée de quelques philosophes touchant le thème de la métaphysique pour le domaine de la philosophie. Notre étude porte uniquement sur la combinatoire française. En conséquence, les documents retenus sont ceux rédigés préférablement en français, d'origines diverses mais de même nature, par des spécialistes pour un public de spécialistes du même domaine. Nous avons constitué notre corpus en numérisant les textes sélectionnés à l'aide d'un lecteur optique pour permettre leur traitement ultérieur par un logiciel de recherche de contextes (Naturel $\mathrm{Pro}^{\mathrm{MC}}$ ). La taille du corpus s'élève à 282849 mots, soit 159373 pour le domaine de l'aéronautique et 
122476 pour le domaine de la philosophie. Nous avons par la suite procédé à la sélection des termes clés par domaine. Pour chacun des domaines, nous avons choisi cinq termes clés qui ne sont pas sémantiquement apparentés (auxquels nous avons associé une étiquette opératoire) et qui dénotent des notions importantes dans les domaines concernés.

Tableau II : Termes clés sélectionnés

\begin{tabular}{|c|c|c|}
\hline DOMAINE & TERME CLÉ & ÉTIQUETTE OPÉRATOIRE \\
\hline Aéronautique & $\begin{array}{l}\text { - aéronef } \\
\text { - aéroport } \\
\text { - piste } \\
\text { - vitesse } \\
\text { - vol }\end{array}$ & $\begin{array}{ll}\varnothing & \text { Entité matérielle } \\
\varnothing & \text { Lieu } \\
\varnothing & \text { Aire physique } \\
\varnothing & \text { Unité de mesure } \\
\emptyset & \text { Activité }\end{array}$ \\
\hline Philosophie & $\begin{array}{l}\text { - amour } \\
\text { - beauté } \\
\text { - connaissance } \\
\text { - être } \\
\text { - vérité }\end{array}$ & $\begin{array}{ll}\emptyset & \text { Disposition } \\
\varnothing & \text { État } \\
\emptyset & \text { Rapports sujet / objet } \\
\varnothing & \text { Entité abstraite } \\
\varnothing & \text { Principe }\end{array}$ \\
\hline
\end{tabular}

La prochaine étape consistait à rechercher des contextes dans le but de sélectionner des combinaisons lexicales représentatives de chacun des domaines. Pour ce faire, nous avons eu recours au logiciel Naturel Pro $^{\mathrm{MC}}$. Ce logiciel est en fait un logiciel de gestion et recherche d'information textuelle. La base documentaire peut être constitué de documents de formats différents, crés par traitement de texte, base de données ou chiffrier électronique. Nous avons choisi de travailler avec ce logiciel, car, grâce à son mode de recherche plein texte et à sa capacité d'indexation positionnelle, il nous a permis d'interroger aisément un ensemble de fichiers et de repérer sans peine des contextes.

Nous avons donc sélectionné les CLS en fonction de la définition que nous avions adoptée : par combinaison lexicale spécialisée, nous entendons toute combinaison de deux lexèmes dont la base (ou noyau) constitue une unité terminologique (simple ou complexe - généralement un nom); les unités lexicales qui cooccurrent avec le terme peuvent appartenir aux catégories nom, verbe ou adjectif; 
et les CLS respectent des conventions d'usage propres à la langue de spécialité concernée.

Cependant, la distinction CLS / terme complexe n'est guère aisée, particulièrement en présence d'associations de types " terme + préposition + nom " ou " nom + préposition + terme $n$. Pour les besoins de notre étude, nous avons décidé de retenir toutes les combinaisons lexicales (y compris celles qui se révélaient des unités terminologiques proprement dites) qui se soumettaient à notre définition, et nous les avons classées par catégories (voir l'annexe 1).

Pour permettre une gestion efficace de l'ensemble des données recueillies nous avons ensuite constitué une base de données informatisée à l'aide du logiciel tableur Excel ${ }^{*}$. La taille de notre corpus étant plutôt restreinte, nous avons choisi de travailler avec ce logiciel en raison de certaines de ses fonctionnalités que nous avons jugées des plus pratiques dans le cadre de notre étude, entre autres la fonction de filtres. En effet, au moyen de ces filtres, nous avons pu retracer aisément et rapidement tous les cooccurrents qui se combinaient avec un terme donné dans les combinaisons particulières recherchées $(V+T$, $T+V$, etc.). L'annexe 2 présente un exemple de notre base de données.

Pour chacun des termes clés, nous avons ensuite effectué une première sélection de cooccurrents dans chacune des catégories, soit cinq (5) dans l'une ou l'autre des catégories verbales $\mathrm{V}+\mathrm{T}$ ou $\mathrm{T}+\mathrm{V}$; cinq (5) dans l'une ou l'autre des catégories nominales $\mathrm{N}+\mathrm{T}$ ou $\mathrm{T}+\mathrm{N}$; et cinq autres dans l'une ou l'autre des combinaisons adjectivales $\mathrm{A}+\mathrm{T}$ ou $T+A$, ce qui totalise 15 cooccurrents par terme clé, pour un ensemble de 75 combinaisons sélectionnées dans chacune des bases. Certains critères ont présidé à la sélection des cooccurrents, soit 1) les cooccurrents ne sont pas synonymes; 2) il existe un seuil d'occurrence minimal; 3) en présence d'un déverbal, non utilisation du verbe correspondant. Le tableau présenté à l'annexe 3 dresse une liste des cooccurrents sélectionnés dans la base aéronautique.

A partir de ces cooccurrents, nous sommes ensuite retournée dans le corpus pour effectuer une deuxième recherche de combinaisons lexicales. Par exemple, pour le terme clé aéronef, nous avons retenu le cooccurrent décoller. Nous sommes donc retournée dans la base de 
textes pour voir si le verbe décoller se combinait avec d'autres termes (comme avion, appareil, etc.). Nous avons relevé tous ces termes de même que la fréquence et les avons consignés dans un autre fichier Excel $^{\Phi}$ (voir un exemple des résultats obtenus à l'annexe 4).

Enfin, à partir des données recueillies au cours de cette deuxième recherche de cooccurrents, nous avons tenté de regrouper les termes possédant des composantes sémantiques communes en nous assurant qu'un seul et unique sens y présidait. Par exemple, pour le terme de départ aéronef, nous avons effectué une deuxième recherche de cooccurrents à partir du verbe exploiter. Parmi les combinaisons relevées, nous avons conservé uniquement les termes pour lesquels le sens de la combinaison correspondait à l'acception suivante : * Faire valoir (une chose); tirer parti de (une chose), en vue d'une production ou dans un but lucratif n. (Le Robert 1995:865). Dans cet exemple, nous avons déterminé l'existence d'au moins deux classes conceptuelles :

1. la classe regroupant les termes qui relèvent du concept $\alpha$ lieu de départ et d'arrivée des moyens de transport aérien ", dans laquelle nous regroupons les termes aérogare, aéroport et infrastructure;

2. la classe regroupant les termes qui relèvent du concept " appareil capable de se déplacer dans les airs ", dans laquelle nous regroupons les termes aéronef, giravion et hélicoptère.

Une fois toutes les étapes de notre processus de recherche effectuées, nous avons pu poursuivre l'analyse proprement dite des résultats afin d'évaluer, entre autres, les différences de comportements des combinaisons lexicales en fonction des domaines de spécialité.

\section{Résultats partiels et conclusion}

Comme nous l'avons mentionné plus haut, notre analyse n'est pas encore terminée. Nous présentons aux annexes 5 et 6 quelques résultats. Nous avons pu dégager certaines combinaisons fortement lexicalisées, indiquant que nous sommes probablement en présence d'unités terminologiques proprement dites. Toutefois, nous n'avons trouvé aucune combinaison que l'on pourrait qualifier de a collocation lexicale ». Mais notre corpus étant tout de même limité, cela n'indique pas l'absence totale de ce type de collocation dans ces domaines spécialisés. Par ailleurs, notre méthode nous a permis de recenser 
différentes catégories de combinaisons et de voir qu'il était tout à fait possible de généraliser les combinaisons lexicales à de plus grands ensembles en regroupant les termes qui possèdent des composantes sémantiques communes (du moins dans le domaine de l'aéronautique). Mais, est-il vrai pour autant, comme l'avance Heid, qu'il existe une prédominance des combinaisons conceptuelles dans les domaines spécialisés? Certes, comme nous venons de l'affirmer, le domaine de l'aéronautique foumit de bons exemples de généralisations possibles. Mais une analyse préliminaire du domaine de la philosophie nous montre que ce dernier est beaucoup moins riche à ce point de vue.

Département de linguistique et traduction Université de Montréal

\section{ANNEXE 1 : CATÉGORIES DE COMBINAISONS LEXICALES}

\begin{tabular}{|l|l|l|}
\hline \multicolumn{1}{|c|}{ CATEGORIES } & \multicolumn{1}{c|}{$\begin{array}{c}\text { EXEMPLES - } \\
\text { AERONAUTIQUE }\end{array}$} & $\begin{array}{c}\text { EXEMPLES - } \\
\text { PHILOSOPHIE }\end{array}$ \\
\hline Combinaison adjectivale & & étre empirique \\
\hline Terme + adjectif & aéroport contrôlé & immuable vérité \\
\hline Adjectif + terme & petit aéroport & \\
\hline
\end{tabular}

\begin{tabular}{|l|l|l|}
\hline Combinaison nominale : & \multicolumn{2}{|l|}{} \\
\hline Terme + préposition + nom & aéroport d'atterrissage & étre de l'ấme \\
\hline Nom + préposition + terme & contrôleur d'aéroport & quête de l'être \\
\hline
\end{tabular}

\begin{tabular}{|l|l|l|}
\hline Combinaison verbale :* & \multicolumn{2}{|l|}{} \\
\hline Terme + verbe & aéronef décolle & étre répond \\
\hline Verbe + terme & maîtriser [un] déronef & justifier l'être \\
\hline
\end{tabular}

* (y compris les combinaisons du type a verbe + joncteur + terme $v$ et terme + joncteur + verbe) 


\section{ANNEXE 2 : EXEMPLE D'ENREGISTREMENTS DE LA BASE_AÉRONAUTIQUE}

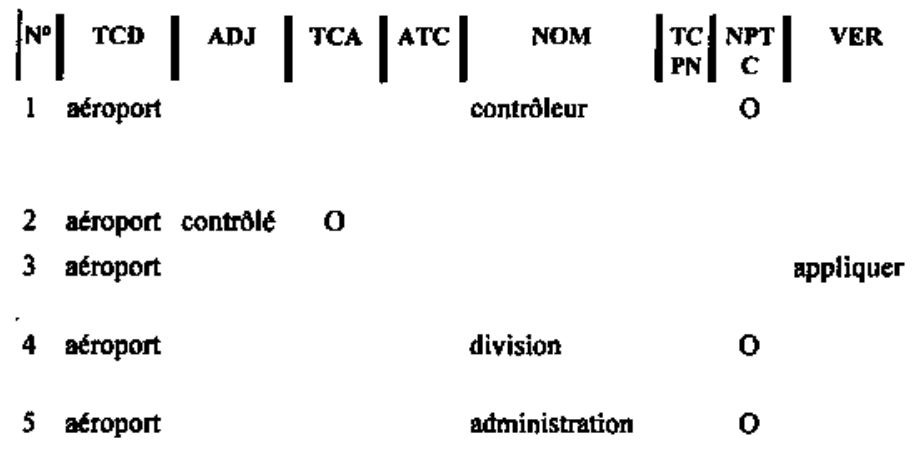

$|\operatorname{Tcv}| \mathbf{v r c} \mid$

Le contröleur d'aéroport peut cependant demander à un pilote d'accuser réception d'un message de façon déterminée

Circuit d'aérodrome aux aéroports contrôlés

o Les procédures suivantes s'appliquent à tous les aéroports

dans beaucoup de villes importantes, il existe une Division des Aéroports

l'administration de l'aéroport est confiée soit au département des Travaux Publics, soit au département des Parcs

Dans cet exemple, nous remarquons les éléments suivants :

$\mathrm{N}^{\mathfrak{b}}$ : le numéro séquentiel de l'entrée; TCD : le terme clé de départ; ADJ : le cooccurrent adjectival du terme clé; TCA : la place qu'occupe le cooccurrent adjectival dans la combinaison, soit Terme clé + Adjectif; ATC : la place qu'occupe le cooccurrent adjectival dans la combinaison, soit Adjectif + Terme clé; NOM : le cooccurrent nominal du terme clé; TCPN : la place qu'occupe le cooccurrent nominal dans la combinaison, soit Terme clé + Préposition + Nom; NPTC : la place qu'occupe le cooccurrent nominal dans la combinaison, soit Nom + Préposition + Terme clé; VER : le cooccurrent verbal du terme clé; TCV : la place qu'occupe le cooccurrent verbal dans la combinaison, soit Terme clé + Verbe; VTC : la place qu'occupe le cooccurrent verbal dans la combinaison, soit Verbe + Terme clé; CTC : le contexte dans lequel se trouve le terme clé. 


\section{ANNEXE 3 : PREMIËRE RECHERCHE DE COOCCURRENTS DANS LA BASE AÉRONAUTIQUE}

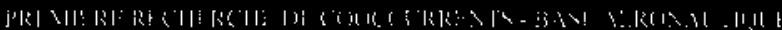

\begin{tabular}{|c|c|c|c|c|c|c|c|c|}
\hline . & 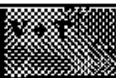 & & 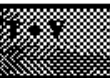 & $x_{x}$ & 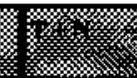 & 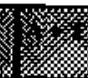 & . & \\
\hline 6 & autoriser & $\mathrm{x}$ & décoller & circulatio & $\begin{array}{l}x \text { départ } \\
\text { x }\end{array}$ & & certifié & $x$ \\
\hline & exploiter & $\mathbf{x}$ & franchir & contróle & $\mathrm{x}$ & & exploité & $\mathbf{x}$ \\
\hline & maîuriser & $x$ & & pilote & $\mathrm{x}$ & & lourd & $x$ \\
\hline & & & & position & $\mathbf{x}$ & & civil & $x$ \\
\hline & & & & & & & IFR & $\mathrm{x}$ \\
\hline 40 & atterrir & $x$ & & Bestion & $\mathrm{x}$ & Grand & contrốlé & $\mathbf{x}$ \\
\hline & implanter & $\mathrm{x}$ & & cout & $\mathrm{x}$ & & $\begin{array}{l}\text { internation } \\
\mathrm{a} 1\end{array}$ & $x$ \\
\hline & desservir & $\mathrm{x}$ & & contrôleur & $x$ & & désignt & $\mathbf{x}$ \\
\hline & fréquenter & $x$ & & activité & $\mathbf{x}$ & & & \\
\hline & intégrer & $x$ & & trafic & $\mathrm{x}$ & & & \\
\hline 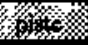 & dégager & $\mathbf{x}$ & & cireuit & $\mathrm{x}$ & & transversal & $\mathbf{x}$ \\
\hline & crojser & $\mathrm{x}$ & & seuil & $\mathbf{x}$ & & prefferentiel & $\mathbf{x}$ \\
\hline & poser (se) & $\mathrm{x}$ & & cap & $\mathbf{x}$ & & actif & $x$ \\
\hline & emprumter & $\mathrm{x}$ & & argle & & & secondaire & $\mathrm{x}$ \\
\hline & survoler & $\mathbf{x}$ & & état & $\mathrm{x}$ & & sec & $\mathrm{x}$ \\
\hline 06 & atteindre & $x$ & angmenter & & croisière & 5 faible & minimal & $x$ \\
\hline & prescrice & $\mathrm{x}$ & & & sécurité & & initial & $x$ \\
\hline & reprendre & $\mathbf{x}$ & & & montée & & Yraie & $\mathrm{x}$ \\
\hline & calculer & $x$ & & & vol & 8 & normalisé & 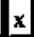 \\
\hline & & & & & descente & & & \\
\hline \% & régejir & $\mathrm{x}$ & traverser & trajextoire & $x$ instruction & & militaire & $x$ \\
\hline & planifier & $x$ & & plan & $x$ & & plané & $x$ \\
\hline & mgintenir & $x$ & & itinéraire & $x$ & & rectiligne & $\mathrm{x}$ \\
\hline & controler & $x$ & & temps & $x$ & & lent & $\mathbf{x}$ \\
\hline & & & & & & & mixte & $\mathbf{x}$ \\
\hline
\end{tabular}


ANNEXE 4 : Deuxième recherche à partir du terme clé * aéronef *

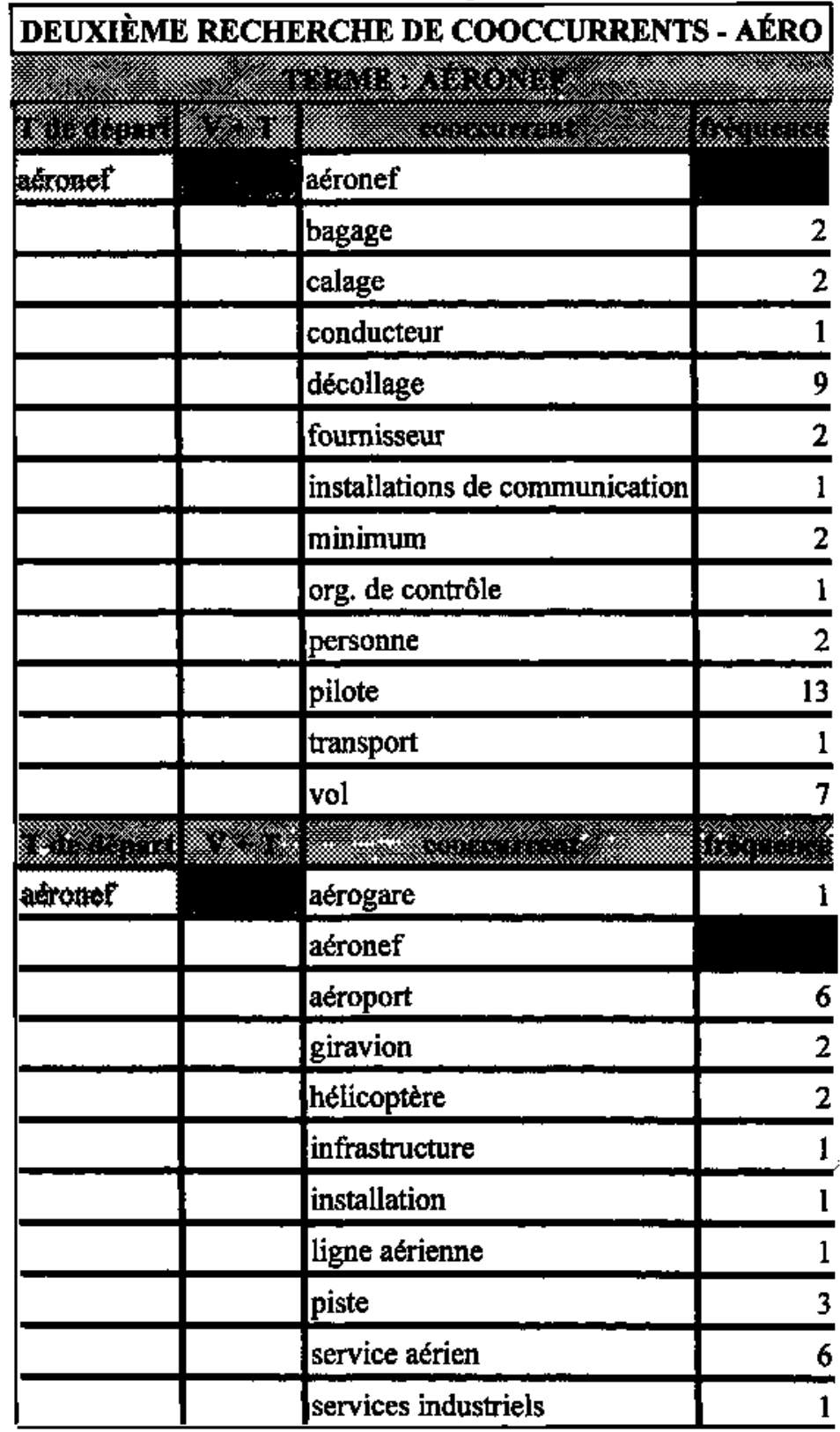




\begin{tabular}{|c|c|c|c|}
\hline & & station radio terrestre & 2 \\
\hline & & vol & 5 \\
\hline (1) & $1: 2$ & 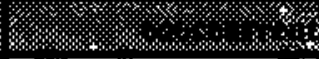 & 18 \\
\hline werouf & & aéronef & \\
\hline & & avion & 7 \\
\hline & & hydravion & 3 \\
\hline & & pilote & 16 \\
\hline & & vol & 2 \\
\hline 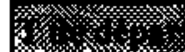 & ; & 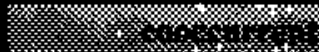 & $4 \times$ \\
\hline méromet & & aéronef & 1 \\
\hline & & appareil & 2 \\
\hline & & avion & \\
\hline & & direction & 2 \\
\hline & & tangage & 2 \\
\hline
\end{tabular}


ANNEXE 5 : Résultats partiels - Domaine aéronautique

\begin{tabular}{|c|c|c|c|c|c|}
\hline $\begin{array}{l}\text { RISSLLL } \\
\text { TFRNIF }\end{array}$ & $\begin{array}{l}\text { TATS A } \\
\text { aComel }\end{array}$ & $\begin{array}{l}\text { LRUA } R=10 \\
\text { acroport }\end{array}$ & piste & & , ol \\
\hline$V+T$ & 158 & 76 & 67 & 80 & 432 \\
\hline $\mathrm{T}+\mathrm{V}$ & 71 & 0 & 0 & 2 & 25 \\
\hline TOTAL_VER & 缕 & 涪, & . & x. & S紋 \\
\hline$N+T$ & 286 & 81 & 195 & 0 & 349 \\
\hline$T+N$ & 77 & 102 & 0 & 804 & 3 \\
\hline TOTAL_NOM & (6) & 48 & 媇 & 好誃 & 然 \\
\hline$A+T$ & 0 & 115 & 0 & 38 & 0 \\
\hline $\mathrm{T}+\mathrm{A}$ & 373 & 191 & 44 & 183 & 132 \\
\hline TOTAL_ADJ & 纹 & 缓 & 椊 & 缨 & 1邺 \\
\hline TOTAL_TERME & 㑫 & s. & 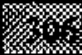 & 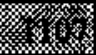 & 的 \\
\hline
\end{tabular}

\section{Résultats_aéronautique}

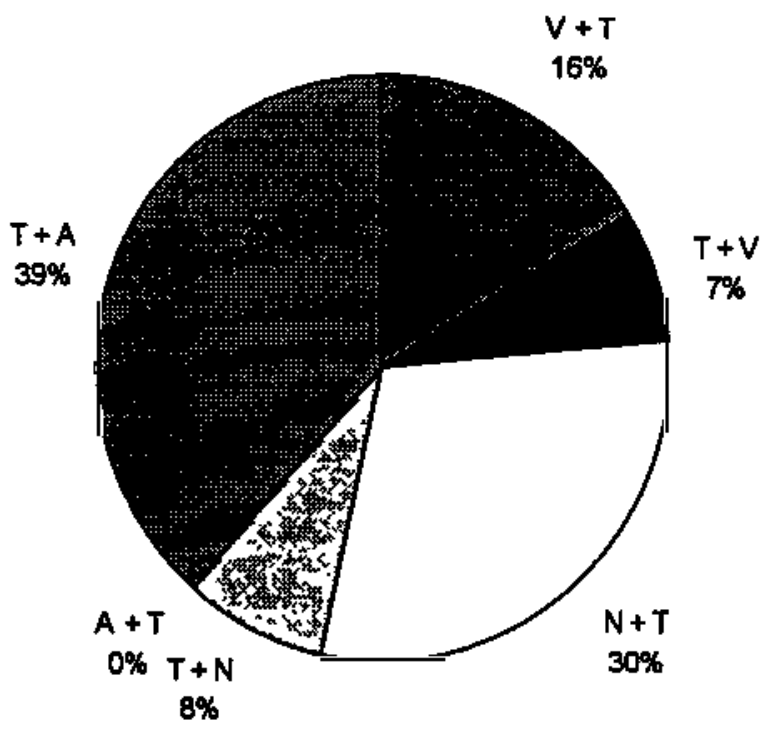


ANNEXE 6 : RÉSULTATS PARTIELS - DOMAINE PHILOSOPHIE

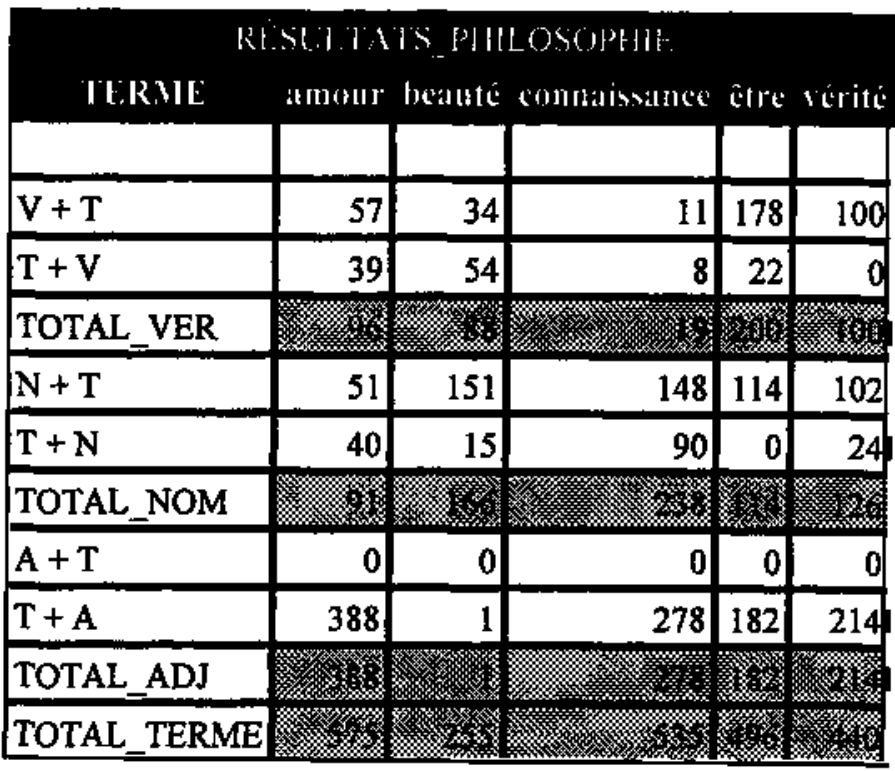

\section{Résultats_philosophie}

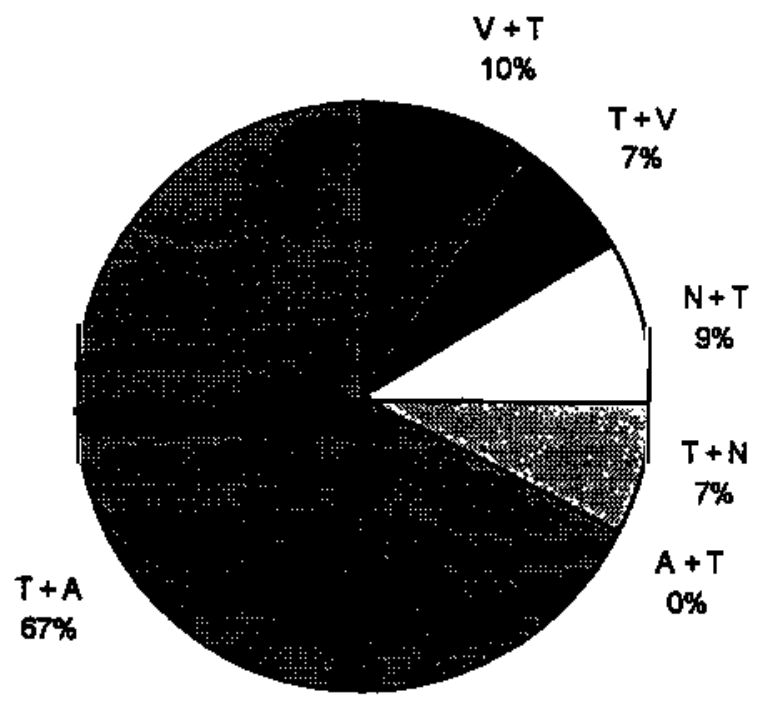




\section{Références}

BENSON, M., BENSON E. et R. ILSON (1997). The BBI Dictionary of English Word Combinations. Revised Edition. Amsterdam/Philadelphia, John Benjamins Publishing Company.

COHEN, B. (1986). Lexique des cooccurrents, bourse - conjoncture économique, Linguatech, $125 \mathrm{p}$.

GROSS, G. (1988). « Degré de figement des noms composés „, Langue française, $\mathrm{n}^{\circ} 90, \mathrm{pp}$. 57-71.

HAUSSMANN, F.J. (1979). * Un dictionnaire des collocations est-il possible?", Travatox de linguistique et de littérature XVII(1), pp. 187195.

HEID, U. (1992). « Décrire les collocations. Deux approches lexicographiques et leur application dans un outil informatisé ", Terminologie et traduction 2/3, pp. 523-548.

HEID, U. (1994). "On the Way Words Work Together - Topics in Lexical Combinatorics ", Martin, W. et al Euralex '94 Proceedings, Amsterdam, pp.226-257.

HEID, U. et G. FREIBOTT (1991). * Collocations dans une base de données terminologique et lexicale *, Meta, 36 (1), mars, pp. 77-91.

KOCOUREK, R. (1982). La langue française de la technique et de la science, Brandstetter Verlag, Wiesbaden, $255 \mathrm{p}$.

L'HOMME, M. C. (1992), * Les unités phraséologiques verbales et leur représentation en terminographie $n$, Terminologie et traduction $2 / 3, \mathrm{pp}$. 493-503.

L'HOMME M. C. (1995). * Processing Word Combinations in Existing Termbanks $n$, Terminology, vol. 2, $n^{\circ} 1$, pp. 141-162.

L'HOMME M. C. et R. GEMME (1997). * Modèle d'accès informatisé aux combinaisons lexicales spécialisées : verbe + nom (terme) et 
extension aux nom (déverbal) + préposition : nom (terme), Lapierre, L., I. Oore et H.R. Runte, Mélanges de linguistique offerts à Rostislav Kocourek, Université Dalhousie (Halifax, Canada), Les Presses ALFA, pp. 89-103.

L'HOMME, M. C. * Caracterisation des combinaisons lexicales spécialisées par rapport aux collocations de langue générale ", Terminologie et dictionnaires (à paraître).

LAINÉ, C., PAVEL, S., BOILEAU, M. (1992). * La phraséologie Nouvelle dimension de la recherche terminologique ", Travaux du module canadien du Rint, L'Actualité terminologique, vol, 25, 3, pp. 59.

MARTIN, W. (1992). "Remarks on Collocations in Sublanguages ", dans Terminologie et traduction, $\mathrm{n}^{\text {os }} 2-3$, pp. 157-164.

MEL'ČUK, I., CLAS, A. et A. POLGUÊRE (1995). Introduction à la lexicologie explicative et combinatoire (ILEC), Éditions Duculot, AUPELF UREF, $256 \mathrm{p}$.

NORME INTERNATIONALE ISO 1087 (1990). Terminologie Vocabulaire.

PAVEL, S. (1993a). « Vers une méthode de recherche phraséologique en langue de spécialité ", L'actualité terminologique 26 (2), pp. 9-13.

PAVEL, S. (1993b). " La phraséologie en langue de spécialité. Méthodologie de consignation dans les vocabulaires terminologiques ", dans Terminologies nouvelles, $\mathrm{n}^{0} 10, \mathrm{pp} .23-35$.

PAVEL, S. (1994). Guide de la recherche phraséologique en langue de spécialité, Bureau de la traduction, pagination discontinue.

ROBERT, P. (1995). Le nouveau Petit Robert, Dictionnaires Le Robert, Paris, 2551 p.

SAGER, J. C. (1990). A Practical Course in Terminology Processing, John Benjamins Publishing Company, Amsterdan / Philadelphia, 249 p. 
RÉSUMÉ : Étude comparative des combinaisons lexicales dans deux domaines du savoir (aéronautique et philosphie) et opposition entre les collocations lexicales et conceptuelles - Notre étude porte sur la combinatoire lexicale en langue de spécialité et cherche a déterminer l'organisation lexicale, syntaxique et sémantique liée à une contrainte d'usage sous-jacente à deux domaines spécifiques - l'aéronautique et la philosophie. Une analyse des points de vue des chercheurs en langue générale et en langue de spécialité sera présentée en première partie. Ensuite, nous ferons la distinction entre les collocations lexicales et les collocations conceptuelles en langue de spécialité. Enfin, nous présenterons la méthodologie utilisée pour mener à bien notre mémoire de maîtrise (en cours) ainsi que des résultats fragmentaires, l'objectif du mémoire étant a) de relever les différents types de combinaisons ( $T+V, V+T, T+\operatorname{adj}$, adj $+T, T+$ prép $+\mathrm{N}, \mathrm{N}+$ prép $+\mathrm{T}$ ); b) de distinguer les collocations lexicales et conceptuelles, s'il en est, et c) d'étudier les tendances de comportement dans l'un et l'autre domaine.

ABSTRACT : Comparative Study of Lexical Combinations in Two Fields of Knowledge (aeronautics and philosophy) and Discrimination Between Lexical Collocations and Conceptual Collocations - This paper deals with word combinations (also called collocations, phraseological units, cooccurrences, etc.) in two different LSPs (Language for Specific Purposes or Special languages) - aeronautics and philosophy. First, a general study of different scientists' approaches in general language and in LSP is provided. Then, the focus is on the discrimination between lexical collocations vs conceptual collocations in LSPs. Finally, an overview of the methodology we used with our master thesis (still in progress) is discussed, along with the presentation of some results.

Claudine Bertrand : département de linguistique et de traduction, Université de Montréal, C. P. 6128, Succursale Centre-Ville, Montréal (Québec) H3C 3J7. Email : bertrac@ere.umontreal.ca 